\title{
The Role of Real-World Evidence in UK Reimbursement: Case Study of Lenalidomide in Myelodysplastic Syndrome Deletion 5q
}

\author{
Dawn Lee ${ }^{1} \cdot$ Nic Brereton ${ }^{1} \cdot$ Sujith Dhanasiri ${ }^{2} \cdot$ Austin Kulasekararaj $^{3}$
}

Published online: 14 December 2018

(c) The Author(s) 2018

\begin{abstract}
Background Uncertainty within cost-effectiveness analysis, often driven by lack of mature data from large clinical trials, plays a key role in decisions made by the National Institute for Health and Care Excellence (NICE), particularly for early access medicines and orphan drugs.

Objectives In this case study, we used real-world evidence to address the uncertainty in the cost-effectiveness case for lenalidomide in transfusion-dependent low- and intermediate-1-risk myelodysplastic syndrome (MDS) deletion 5q [del(5q)], affecting a small but unique subpopulation with an orphan disease.

Methods As part of a submission to NICE, we developed a cost-effectiveness model for lenalidomide, resulting in eventual recommendation.

Results Due to data limitations within the trial evidence available, the model was based on surrogate outcomes supported by a disease-wide literature review. The validity of modelled estimates for critical long-term outcomes in terms of time on treatment (32\% reaching 26 cycles when the patient access scheme applied in the model vs. $28 \%$ in the real-world data) and survival was confirmed using real-world evidence (projected median survival for best supportive care of 4.3 years vs. real-world evidence showing median survival with low- and intermediate-1-risk MDS of 5.7 and 3.5 years, respectively). Conclusion This case study demonstrates the usefulness and relevance of the application of real-world data when trial data are limited.
\end{abstract}

\section{Key Points for Decision Makers}

Based on this case study, we recommend that the search for real-world evidence is initiated prior to submission of technologies to health technology assessment authorities.

A willingness to provide reassurance on outcomes with future data collection may reduce delays in new therapy reimbursement.

Electronic supplementary material The online version of this article (https://doi.org/10.1007/s41669-018-0110-3) contains supplementary material, which is available to authorized users.

Dawn Lee

dlee@bresmed.com

1 BresMed, North Church House, 84 Queen Street, Sheffield S1 2DW, UK

2 Celgene International, Boudry, Switzerland

3 King's College Hospital National Health Service Foundation Trust, London, UK

\section{Introduction}

Reimbursement by the National Institute for Health and Care Excellence (NICE) is a key determinant for global market access. Uncertainty within cost-effectiveness analysis, which is often driven by a lack of mature data from large clinical trials, plays a key role in decisions made by NICE, particularly for early access medicines and orphan drugs.

Real-world evidence on the impact of current care treatments on patient outcomes is often available from published literature and registries. This information is also sometimes available where early access programmes are in place.

In this case study, we used real-world evidence to address the uncertainty in the cost-effectiveness case for lenalidomide in transfusion-dependent low- and intermediate-1-risk myelodysplastic syndrome (MDS) deletion 5q [del(5q)], which affects a small but unique subpopulation of patients with an orphan disease.

Lenalidomide (Revlimid ${ }^{\circledR}$, a registered trademark of Celgene Corporation) is an oral therapy that modifies the natural course of MDS through clonal suppression, which 
delays disease progression, thereby reducing the need for blood transfusions. Marketing authorisation was granted in Europe on 14 June 2007 for the treatment of patients when other therapeutic options are insufficient or inadequate [1].

Available trial data comprise one randomised controlled trial (RCT) (MDS-004: $n=205$; maximum duration of follow-up: 3 years), with immature follow-up in the context of lifetime cost-effectiveness analysis and a very high level of early crossover in the placebo arm (only one patient in the placebo group completed the 52-week double-blind phase), and one phase II trial (MDS-003). This prevents analysis of long-term outcomes by treatment received and makes it difficult to implement the patient access scheme (PAS) for lenalidomide in the UK, which is a scheme in which treatment is given for free after 26 cycles and therefore relies on an accurate estimation of the proportion of patients still on treatment at 26 cycles.

We developed a cost-effectiveness model for lenalidomide based on surrogate outcomes supported by a diseasewide literature review and validated the critical long-term outcomes with UK real-world evidence as part of the NICE appraisal process. This article provides the details of this case study, demonstrating how real-world evidence can be used to supplement and inform modelling. Real-world evidence was used to validate outcomes for time on treatment (and, critically, the proportion of patients reaching 26 cycles, which predicts the benefit to the National Health Service [NHS] from free treatment from this point onwards), the use of transfusion dependency as a surrogate for overall survival (OS), long-term predictions of OS and the generalisability of the patient population in MDS-004.

\section{Methods}

\subsection{Selection of Model Surrogate Outcomes for Myelodysplastic Syndrome}

MDS is a group of disorders caused by insufficient numbers of mature blood cells being produced [2]; therefore, treatment regimens can involve hospitalisation for receipt of chemotherapy drugs and/or antibiotics and regular blood transfusions [3-6]. Red blood cells (RBCs), white blood cells and platelets may all be affected by MDS, and failure to treat MDS can result in life-threatening complications (infections and bleeding), disease transformation to acute myeloid leukaemia (AML) and impaired quality of life. However, the clinical and economic burden of MDS increases quickly due to the cost of frequent transfusions and the administration of chelating agents (in centres where this is available) to remove iron accumulation as a consequence of transfusions [7-10]. Additionally, the vast majority (> 70\%) of patients with MDS are 'low risk' and elderly, necessitating optimal management of co-morbidities to ensure a good quality of life.

Reducing the number of patients who are transfusion dependent can therefore lead to the following benefits:

- Reduction in the demand for transfusion and associated healthcare-related costs

- Reduction in the cost of transfusion-related chelation therapy

- Potential reduction in the cost of managing cardiovascular disease, diabetes mellitus and hepatic complications related to anaemia and iron overload

- Improved survival

- Improved patient quality of life while on treatment (symptomatic) and beyond (through delaying the onset of co-morbidities and disease progression in responding patients).

By reducing dependency on blood transfusions, lenalidomide could prevent the accelerating morbidity, mortality and quality-of-life impairment associated with MDS disease progression.

To validate the conceptual model, a systematic literature review was conducted to determine the strength of the relationship between transfusion dependency and both survival and AML progression.

\subsection{Model Structure}

Based on the expected importance of transfusion dependency to patients and the healthcare system, we developed a Markov cost-effectiveness model with health states defined by transfusion dependency, the consequences of dependency (such as iron chelation and associated complications), AML and survival (Fig. 1). The model was constructed in Microsoft Excel ${ }^{\circledR}$ (Microsoft Corp., Redmond, WA, USA), primarily using MDS-004 clinical trial data from the intention-totreat population [11] and was based on a 20-year (lifetime) time horizon. All costs and benefits were discounted at 3.5\% over a lifetime horizon, in line with UK guidelines $[12,13]$. The model took a UK NHS perspective using a 2011/2012 cost year, which was the latest available cost information at the time of construction.

For further information on the model structure and a list of the model input parameters, please see Tables S1 and S2 in the Electronic Supplementary Material.

\subsection{Real-World Evidence Used for Validation}

Time on treatment and the generalisability of the patient population in MDS-004 to clinical practice were validated using data from a UK database of all patients who receive lenalidomide. The Treatment Continuation Scheme (TCS) 


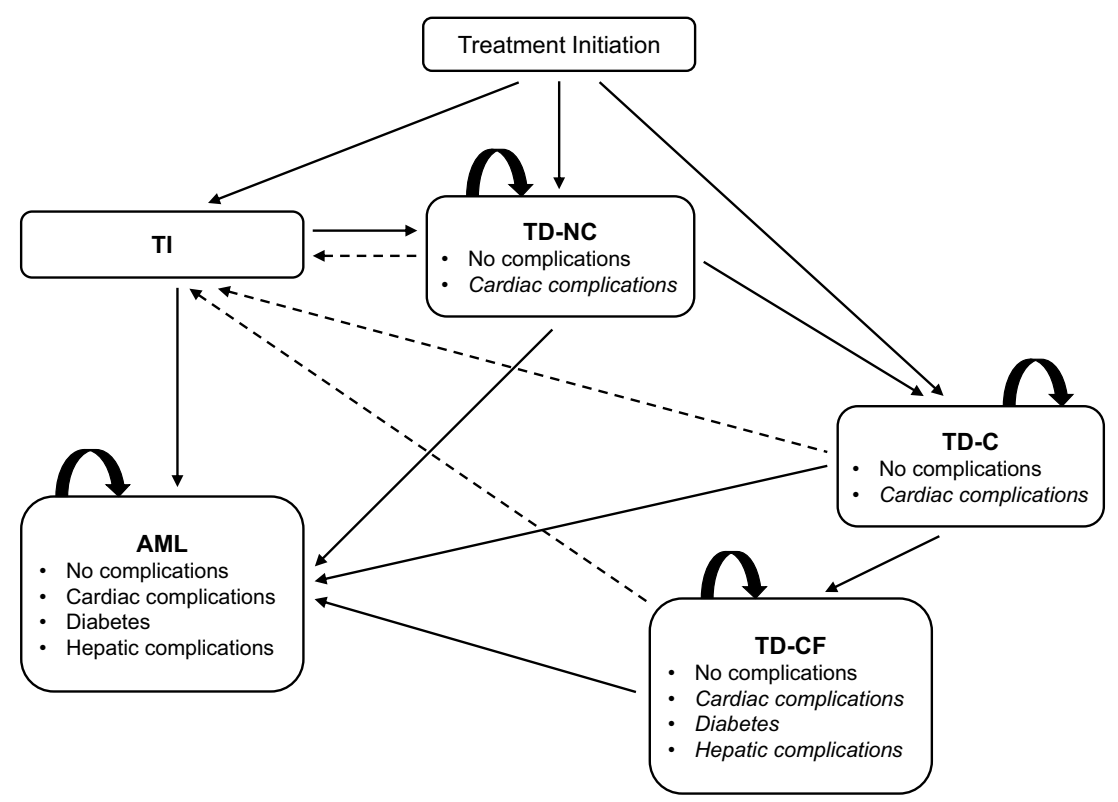

Fig. 1 Markov model structure. Patients can become transfusion independent due to erythropoiesis-stimulating agent + granulocyte colony-stimulating factor (represented by dashed arrows). Cardiac complications can occur in any of the transfusion-dependent states, and diabetes mellitus and hepatic complications can occur in the TD-CF state (indicated by italicised text). Once complications have been experienced, they remain for the rest of the patient's life. The absorbing state is death. The cycle length and time horizon are 28 days and 20 years, respectively. $A M L$ acute myeloid leukaemia, $C$ chelation, $C F$ chelation failure, $N C$ no chelation, $T I$ transfusion independence, $T D$ transfusion dependence

Database was set up for monitoring for the Pregnancy Prevention Programme (PPP) in the UK, which is a regulatory requirement given the teratogenic nature of lenalidomide. The database is also used to administer the PAS currently in place in England for lenalidomide for the treatment of multiple myeloma in patients who have received at least one prior therapy. Lenalidomide cannot be prescribed without a prescription authorisation form (PAF) signed by a physician. All PAFs for lenalidomide are logged into the database regardless of the indication to monitor and assist with the PPP. Patients were able to access lenalidomide prior to NICE approval free of charge via individual prescriber requests, which meant that data were available on historical use for a limited number of outcomes.

Data were extracted from the TCS Database to analyse the time spent on treatment of patients with MDS who received lenalidomide between 5 February 2009 and 1 February 2014. Patient-level data detailed information on patients' age, sex, the number of cycles (of 28 days) for which they were on treatment, and whether they were still on active treatment on 1 February 2014.

Kaplan-Meier methodology was used to evaluate the time on treatment data: patients who had discontinued treatment were classed as experiencing an event, irrespective of the reason for discontinuation. Patients were also classed as having discontinued treatment if their last PAF received by Celgene Corporation was more than 6 months before 1 February
2014, as historically PAFs were usually received with a lag time of between 3 and 6 months. Although PAFs are occasionally received beyond 6 months, this is not standard practice. If a PAF was received within the 6 months before 1 February 2014, the patient was classed as remaining on treatment and therefore censored.

The following patient details were available. Records were complete for all patients included in the database:

- Total number of patients: 124

- Average age at start of treatment: 70.85 years

- Female: $68.5 \%$

- Of these, $4.7 \%$ had childbearing potential

- Number of patients still receiving treatment on 1 February 2014: 50.

It is important to note that TCS data could, in theory, include MDS del(5q) patients with a complex karyotype as well as non-del(5q) patients, both of which are known to have lower response rates and a lower duration of RBC transfusion independence than del(5q) patients [14]. Additionally, there is a higher likelihood of patients being treated for more advanced disease because lenalidomide was not licensed at the time of starting treatment for many patients included in this dataset. Off-label/compassionate use is often reserved for those patients with the greatest need; for example, those patients whom a physician believes will 
progress more rapidly or who have a higher transfusion burden, indicating a more aggressive disease. Therefore, this analysis is likely to underestimate the duration of treatment that could be achieved within the isolated del( $5 q)$ licence of lenalidomide.

OS outcomes were validated using published information from 1000 newly diagnosed MDS patients with low- and intermediate-1-risk MDS from the European Leukemianet MDS (EUMDS) Registry [15] and older published information from the International MDS Risk Analysis Workshop, which combined clinical data from seven large previously reported risk-based studies $(n=816)$ that had generated prognostic systems [16]. These were selected as they were the largest available data sources for validation and because the UK TCS data did not contain information on OS. EUMDS represents the more relevant source being both EU data and more recent. Medians and Kaplan-Meier curve fits were compared based on a weighted average of the lowand intermediate-1-risk populations using the proportions from the MDS-004 trial (48.9\% vs. $51.1 \%$, respectively). Modelled data were considered a good fit if the registry fell within the $95 \%$ confidence interval (CI) of the modelled estimates. Assessment against registry CIs could not be conducted as this information was not reported in the original publications.

\section{Results}

\subsection{Evidence of Surrogacy Between Transfusion Dependence and Survival}

Evidence from the systematic literature review and the MDS-004 clinical trial demonstrated a strong relationship between transfusion dependency and survival, and a weak relationship between transfusion dependency and AML [11, 17]. Transfusion dependence in patients with MDS was therefore an appropriate selection as a surrogate outcome in the model.

In a landmark 6-month analysis, the MDS-004 study showed significantly improved survival prognoses in lowand intermediate-1-risk patients with isolated del(5q) who experienced a period of at least 182 days of transfusion independency (response) compared with those who did not [18]. Median OS was 5.7 years (95\% CI 3.2-not reported) in responders, compared with just 2.7 years (95\% CI 1.5-4.8; $p=0.0072$ ) in non-responders. This is consistent with outcomes in the phase II MDS-003 study, which showed a significantly longer median OS in patients achieving response (4.3 vs. 2.0 years in non-responders; $p<0.0001$ ) [19].

The systematic literature review and meta-analysis conducted by the University of Sheffield School of Health and Related Research (ScHARR) in 2014 provided additional evidence for the importance of transfusion dependency as a prognostic factor [17]. Based on ten studies, a pooled effect of transfusion independency on OS was characterised by a hazard ratio (HR) of 0.41 (95\% CI 0.29-0.56), equating to a $59 \%$ decrease in the risk of death compared with transfusion-dependent patients in the overall MDS population. For low-risk patients with MDS, the HR for transfusion independency relative to dependency was estimated to be 0.53 (95\% CI 0.27-1.05), which means a 47\% decrease in the risk of death. Study overlap prevented a meta-analysis of studies where patients entered as transfusion dependent and became transfusion independent during the course of the study. However, these studies did report HRs ranging from 0.53 to 0.36 (a mortality reduction ranging from 47 to $64 \%$ for transfusion independence). These estimates are consistent with the results reported within the MDS-004 trial $(\mathrm{HR}=0.53$; 95\% CI 0.31-0.91) [18].

Evidence of the link between AML and transfusion dependency was less conclusive. In a multivariate analysis, transfusion need was identified as a risk factor for AML progression [8]. However, in the 6-month landmark analysis, the probability of AML progression was not significantly different between responders (RBC transfusion independent for $\geq 182$ days) and non-responders (log-rank test $p=0.9883$ ), with 2-year AML progression rates of $7.3 \%$ (95\% CI 2.4-21.1) and 15.5\% (95\% CI 6.7-33.6), respectively [18]. Therefore, despite the numerical advantage, the final base-case model assumed equal rates of AML progression for transfusion-dependent and -independent patients.

\subsection{Comparison of Patient Demographics}

The demographics of patients included within the MDS-004 trial were broadly consistent with those receiving lenalidomide in the TCS Database (the average age at start of treatment was 68 vs. 71 years, respectively; the proportion of female patients was $76 \%$ vs. $69 \%$, respectively).

\subsection{Cost Effectiveness}

Lenalidomide was cost effective at the UK willingnessto-pay threshold of $£ 30,000$ per quality-adjusted life-year (QALY), with an incremental cost-effectiveness ratio (ICER) of $£ 25,310$ in the base-case economic analysis (Table 1). The primary source of QALY benefit came from extended time in transfusion independence, with an additional 0.72 QALYs (discounted) gained by patients receiving lenalidomide (1.33 additional QALYs in the response state). The primary source of cost offset came from reduced chelation and number of transfusions, with savings of $£ 14,313$ and $£ 11,669$, respectively, over a patient's lifetime. The key uncertainties identified through a sensitivity analysis were the time horizon used for the analysis, the proportion of patients who reached 
Table 1 Results of base-case analysis

\begin{tabular}{|c|c|c|c|}
\hline Base-case analysis & Best supportive care & Lenalidomide & Net change \\
\hline \multicolumn{4}{|l|}{ Costs (discounted) $[£]$} \\
\hline Cost of treatment (drug treatment and transfusions) & 2243 & 43,359 & 41,116 \\
\hline Adverse events associated with treatment & 0 & 184 & 184 \\
\hline Cost of iron chelation & 45,753 & 31,440 & $-14,313$ \\
\hline Cost of transfusion and chelation complications & 958 & 956 & -2 \\
\hline Cost of blood transfusion & 61,083 & 49,413 & $-11,669$ \\
\hline Cost of AML & 3871 & 4558 & 688 \\
\hline General monitoring costs & 84 & 4553 & 4469 \\
\hline Monitoring cost with standard care & 9208 & 6977 & -2230 \\
\hline Monitoring cost for iron chelation & 43 & 29 & -13 \\
\hline Total cost & 123,241 & 141,470 & 18,229 \\
\hline \multicolumn{4}{|l|}{ Life-years (undiscounted) } \\
\hline $\mathrm{TI}$ & 0.15 & 1.72 & 1.57 \\
\hline TD_no chelation & 0.13 & 0.26 & 0.13 \\
\hline TD—chelation & 3.00 & 2.17 & -0.83 \\
\hline TD_chelation failure & 1.66 & 1.61 & -0.04 \\
\hline AML & 0.24 & 0.29 & 0.05 \\
\hline Total life-years & 5.17 & 6.06 & 0.88 \\
\hline \multicolumn{4}{|l|}{ QALYs (discounted) } \\
\hline TI & 0.12 & 1.35 & 1.23 \\
\hline TD—no chelation & 0.08 & 0.16 & 0.08 \\
\hline TD—chelation & 1.70 & 1.16 & -0.54 \\
\hline TD_chelation failure & 0.92 & 0.85 & -0.07 \\
\hline AML & 0.13 & 0.15 & 0.02 \\
\hline Total QALYs & 2.95 & 3.67 & 0.72 \\
\hline \multicolumn{4}{|l|}{ Incremental cost-effectiveness ratios } \\
\hline Cost per life-year gained & & & $£ 20,639$ \\
\hline Cost per QALY gained & & & $£ 25,310$ \\
\hline
\end{tabular}

$A M L$ acute myeloid leukaemia, $Q A L Y$ quality-adjusted life year, $T D$ transfusion dependent, $T I$ transfusion independent
26 cycles and the survival assumed for best supportive care (BSC) (Tables S1 and S2; Electronic Supplementary Material). Assuming a time horizon of more than 5 years, lenalidomide is cost effective versus BSC (ICER $=£ 30,923$ at 5 years). Based on threshold analysis, provided that $27 \%$ of patients reach 26 cycles of treatment, lenalidomide remains cost effective at a threshold of $£ 30,000$ per QALY.

Probabilistic sensitivity analysis showed a mean ICER similar to the deterministic case ( $£ 25,708)$, with a reasonably large amount of uncertainty in both costs and QALYs; this reflects the limited amount of data available for analysis in this orphan indication (Fig. 2). In $65 \%$ of scenarios, lenalidomide was cost effective at a willingness-to-pay threshold of $£ 30,000$ per QALY, rising to $99 \%$ at a threshold of $£ 50,000$ (Fig. S1; Electronic Supplementary Material).

NICE viewed the remaining uncertainties as acceptable provided that Celgene Corporation agreed to collect additional data on long-term outcomes following recommendation.

\subsection{Validation of Time on Treatment}

Median time on treatment was somewhat shorter in the TCS Database than the modelled projections, but the projections did fall within the $95 \%$ CI (eight cycles [95\% CI 4-14] vs. 11 cycles). The restricted mean for the TCS dataset was more consistent at 19.7 cycles (95\% CI 14.7-24.7) versus the modelled 21.4 cycles (mean restricted to 69 cycles, which was the maximum observed cycles within the TCS data). The proportion of patients expected to reach 26 cycles on lenalidomide, and therefore benefit from the PAS, was also consistent across the extrapolation from the MDS-004 trial and the real-world evidence from the TCS Database ( $32 \%$ vs. $28 \%$ [95\% CI $0.20-0.38$ ]; Fig. 3). This is in line with what was found through both clinical opinion gained via consultation with experts as part of the NICE submission process and evidence from the USA. US real-world data indicated that usage of lenalidomide in patients with MDS drops steeply in the 
Fig. 2 Cost-effectiveness plane. Observations shown are the results of probabilistic sensitivity analysis of the Markov model of lenalidomide in myelodysplastic syndrome deletion $5 \mathrm{q}[\operatorname{del}(5 \mathrm{q})]$, with WTP thresholds of $£ 20,000$ and $£ 30,000$ displayed for reference. $Q A L Y$ quality-adjusted life-year, $W T P$ willingness to pay
Fig. 3 Time on treatment of patients receiving lenalidomide for myelodysplastic syndrome deletion $5 \mathrm{q}[\operatorname{del}(5 \mathrm{q})]$; clinical trial results versus UK TCS results. TCS Treatment Continuation Scheme Database
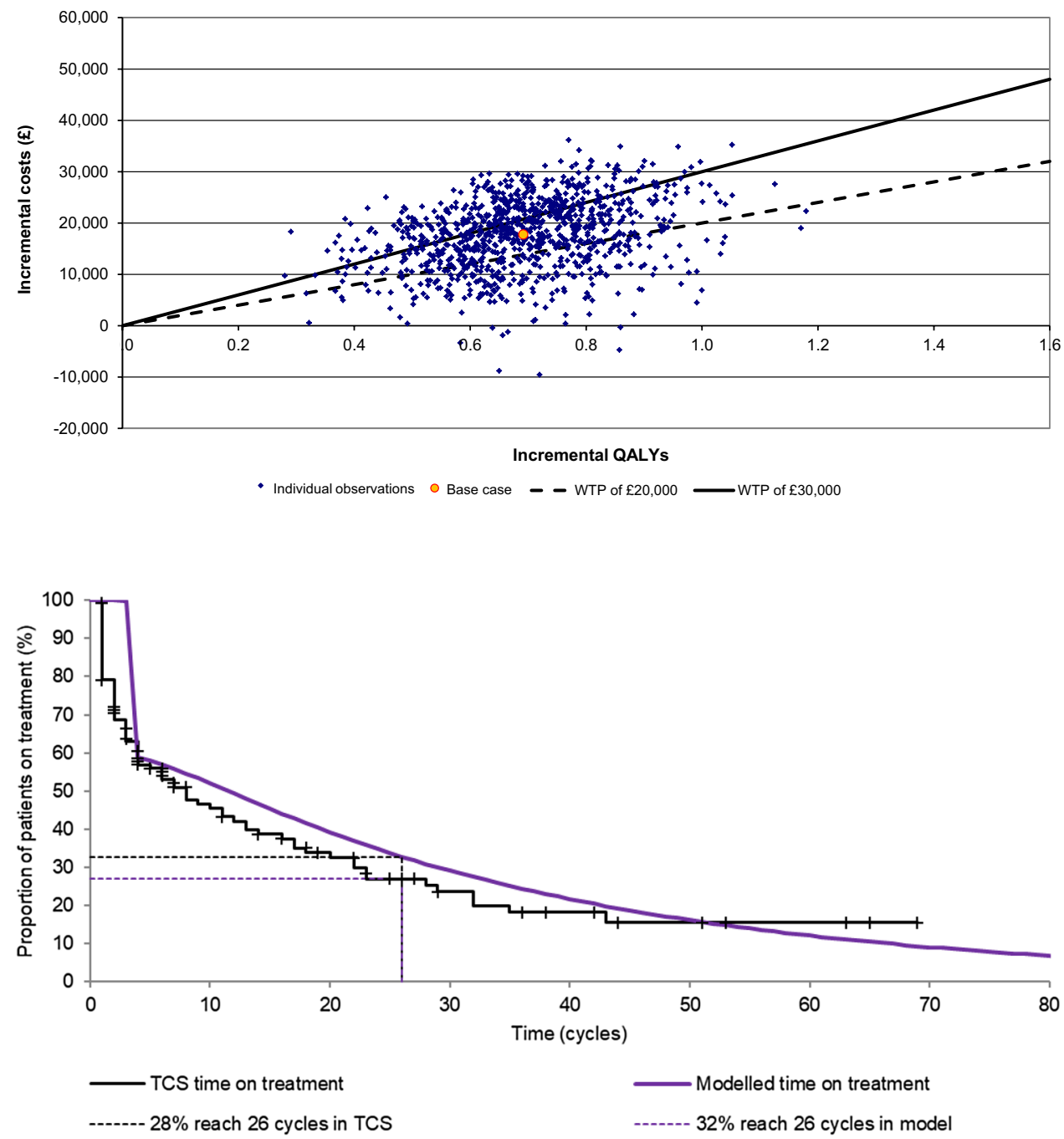

first three cycles, with continuation of treatment for at least three cycles being linked to an increased chance of improved outcome in terms of response [20].

\subsection{Validation of Survival Outcomes}

Based on the modelled extrapolation of the clinical trial data, patients receiving lenalidomide are expected to survive on average 0.9 years longer than those receiving UK BSC (6.1 vs. 5.2 years, undiscounted) (Figs. S2-S4; Electronic Supplementary material).

The model was checked for internal and external consistency. Internal consistency was maintained as the median OS predicted by the model was reflective of the results of the lenalidomide clinical trials (modelled median for lenalidomide: 5.1 vs. 5.2 years in the MDS-003 and MDS-004 clinical trials, respectively). Projected median survival for BSC (4.3 years [95\% CI 2.9-5.0]) was also consistent with external data:
- Greenberg et al. [16] indicated that median survival with low- and intermediate-1-risk MDS is 5.7 and 3.5 years, respectively, with a weighted average using MDS-004 risk-level data of 4.6. The weighted average based on de Swart et al. [15] is 4.0.

- Digitised Kaplan-Meier estimates of OS weighted by risk status from EUMDS and Greenberg et al. [16] (Fig. S5; Electronic Supplementary Material) showed that model projections were consistent with EUMDS (the more recent of the sources), with projections long-term falling below those from Greenberg et al. [16] (although it should be noted that this is the more historical and less relevant of the two sources).

\section{Discussion}

This case study demonstrates the usefulness and relevance of the application of real-world data when trial data are limited. The use of real-world evidence to validate 
outcomes both prior to recommendation and post acceptance is becoming increasingly popular, with similar examples seen in recent melanoma submissions to NICE since the recommendation for lenalidomide [21]. The role of real-world evidence in decision-making has recently been a topic of considerable discussion. While real-world evidence can be subject to bias, it plays an important role in demonstrating the effectiveness of treatments in clinical practice and can therefore play a key part in addressing uncertainties in the translation of RCT data into a realworld setting.

The role of uncertainty in the decision-making process of NICE is clear. A large number of technology appraisals proceed through multiple rounds of appraisal, requiring additional analysis to address key uncertainties identified by NICE. It is insufficient to simply demonstrate a plausible ICER within the usual willingness-to-pay thresholds; the ICER needs to be shown to be robust. This requirement naturally increases the difficulty of obtaining reimbursement in areas where few data are available, such as orphan and paediatric indications and drugs granted rapid approval by the European Medicines Agency.

The weakness of this analysis lies in the limited amount of data available in terms of patient numbers, length of follow-up and availability of data for BSC. This limitation only increases the importance of using external datasets, both to demonstrate the validity of surrogate outcome relationships and to confirm expected outcomes in clinical practice.

The availability of real-world data on the use of lenalidomide, although limited, is also perhaps unusual as the majority of treatments being assessed for reimbursement would not have generated sufficient evidence to conduct long-term validation via early access or compassionate use schemes. This limits the generalisability of this aspect of the analysis to other treatments.

Based on this case study, we would recommend that the search for such evidence should be initiated prior to submission to health technology assessment authorities. Additionally, a willingness to provide reassurance on outcomes with future data collection could lead to reduced delays in the reimbursement of new medications, which is increasingly important since NICE took over responsibility for Cancer Drugs Fund spending in 2016.

\section{Conclusion}

Our analysis demonstrates the cost effectiveness of lenalidomide for the treatment of MDS and that, with the correct use of real-world evidence, reimbursement can be obtained for orphan disease treatments such as lenalidomide where RCT evidence is inherently limited.
Author Contributions DL performed analyses and wrote the manuscript. NB performed analyses and provided draft assistance during preparation of this manuscript. SD and AK contributed to writing and editing the manuscript. DL will act as the overall guarantor. All authors have read and approved the final version of the manuscript.

\section{Compliance with Ethical Standards}

Conflict of interest Dawn Lee and Nic Brereton are employed by BresMed Health Solutions Ltd, a consultancy company that was commissioned by Celgene Corporation to perform the reported analysis. Sujith Dhanasiri is an employee of Celgene Corporation, which funded this study. Austin Kulasekararaj received honorarium, consulting fees and payment for lectures and educational meetings from Celgene Corporation.

Funding Celgene Corporation provided funding for this study.

Data availability statement Data requests may be submitted to Celgene at http://www.CelgeneClinicalDataSharing.com and must include a description of the research proposal.

Open Access This article is distributed under the terms of the Creative Commons Attribution-NonCommercial 4.0 International License (http://creativecommons.org/licenses/by-nc/4.0/), which permits any noncommercial use, distribution, and reproduction in any medium, provided you give appropriate credit to the original author(s) and the source, provide a link to the Creative Commons license, and indicate if changes were made.

\section{References}

1. Celgene Ltd. Revlimid ${ }^{\circledR}$. Summary of product characteristics. 2014. http://www.medicines.org.uk/emc/medicine/19841/SPC/ Revlimid. Accessed 13 Jan 2015.

2. Besa EC. Myelodysplastic syndromes (refractory anemia). A perspective of the biologic, clinical, and therapeutic issues. Med Clin North Am. 1992;76(3):599-617.

3. List AF, Vardiman J, Issa JP, DeWitte TM. Myelodysplastic syndromes. Hematology Am Soc Hematol Educ Program. 2004:297-317.

4. Balducci L. Transfusion independence in patients with myelodysplastic syndromes: impact on outcomes and quality of life. Cancer. 2006;106(10):2087-94.

5. Mallo M, Cervera J, Schanz J, Such E, Garcia-Manero G, Luño E, et al. Impact of adjunct cytogenetic abnormalities for prognostic stratification in patients with myelodysplastic syndrome and deletion 5q. Leukemia. 2011;25(1):110-20.

6. Oliva EN, Schey C, Hutchings AS. A review of anemia as a cardiovascular risk factor in patients with myelodysplastic syndromes. Am J Blood Res. 2011;1(2):160.

7. Frytak JR, Henk HJ, De Castro CM, Halpern R, Nelson M. Estimation of economic costs associated with transfusion dependence in adults with MDS. Curr Med Res Opin. 2009;25(8):1941-51.

8. Malcovati L, Della Porta MG, Strupp C, Ambaglio I, Kuendgen A, Nachtkamp K, et al. Impact of the degree of anemia on the outcome of patients with myelodysplastic syndrome and its integration into the WHO classification-based Prognostic Scoring System (WPSS). Haematologica. 2011;96(10):1433-40. 
9. Lyman GH, Berndt ER, Kallich JD, Erder MH, Crown WH, Long $\mathrm{SR}$, et al. The economic burden of anemia in cancer patients receiving chemotherapy. Value Health. 2005;8(2):149-56.

10. Varney SJ, Guest JF. The annual cost of blood transfusions in the UK. Transfus Med. 2003;13(4):205-18.

11. Fenaux P, Giagounidis A, Selleslag D, Beyne-Rauzy O, Mufti $\mathrm{G}$, Mittelman M, et al. A randomized phase 3 study of lenalidomide versus placebo in RBC transfusion-dependent patients with Low-/Intermediate-1-risk myelodysplastic syndromes with del5q. Blood. 2011;118(14):3765-76.

12. National Institute for Health and Care Excellence. Guide to the methods of technology appraisal. 2013. https://www.nice.org.uk/ process/pmg9/chapter/foreword. Accessed 6 Jan 2016.

13. HM Treasury. The green book. Central government guidance on appraisal and evaluation. OGL; 2018.

14. Raza A, Reeves JA, Feldman EJ, Dewald GW, Bennett JM, Deeg HJ, et al. Phase 2 study of lenalidomide in transfusiondependent, low-risk, and intermediate-1-risk myelodysplastic syndromes with karyotypes other than deletion 5q. Blood. 2008;111(1):86-93.

15. De Swart L, Smith A, Johnston T, Haase D, Droste J, Fenaux P, et al. Validation of the Revised International Prognostic Scoring System (IPSS-R) in 1000 newly diagnosed MDS patients with Low- and Intermediate-1 risk MDS in the European Leukemianet MDS (EUMDS) Registry. Blood. 2013;122(21):2770 (Abstract).
16. Greenberg P, Cox C, LeBeau MM, Fenaux P, Morel P, Sanz G, et al. International scoring system for evaluating prognosis in myelodysplastic syndromes. Blood. 1997;89(6):2079-88.

17. Harnan S, Ren S, Gomersall T, Everson-Hock ES, Sutton A, Dhanasiri S, et al. Association between transfusion status and overall survival in patients with myelodysplastic syndromes: a systematic literature review and meta-analysis. Acta Haematol. 2016;136(1):23-42.

18. Giagounidis A, Mufti GJ, Mittelman M, Sanz G, Platzbecker U, Muus $\mathrm{P}$, et al. Outcomes in RBC transfusion-dependent patients with Low-/Intermediate-1-risk myelodysplastic syndromes with isolated deletion $5 \mathrm{q}$ treated with lenalidomide: a subset analysis from the MDS-004 study. Eur J Haematol. 2014;93(5):429-38.

19. List AF, Bennett JM, Sekeres MA, Skikne B, Fu T, Shammo JM, et al. Extended survival and reduced risk of AML progression in erythroid-responsive lenalidomide-treated patients with lower-risk del(5q) MDS. Leukemia. 2014;28(5):1033-40.

20. Zeidan AM, Gore SD, McNally DL, Baer MR, Hendrick F, Mahmoud D, et al. Lenalidomide performance in the real world: patterns of use and effectiveness in a Medicare population with myelodysplastic syndromes. Cancer. 2013;119(21):3870-8.

21. National Institute for Health and Care Excellence. TA319: Ipilimumab for previously untreated advanced (unresectable or metastatic) melanoma. 2014. https://www.nice.org.uk/guidance/ta319. Accessed 8 Jan 2015 